\title{
Effect of allelic forms of GRFs genes on the development of common wheat under different conditions of nitrogen supplementation
}

\author{
Divashuk M.G.*, Litvinov D.Y., Chernook A.G., Karlov G.I., Bazhenov M.S. \\ All-Russia Research Institute Of Agricultural Biotechnology, Moscow, Russia \\ * email:divashuk@gmail.com
}

Nitrogen fertilizers increase the yield of cereals, but at the same time they increase the height of the plants and make the plants susceptible to lodging. To improve resistance to lodging, "dwarfing" genes are employed, however in addition to reducing plant height, these genes reduce the response to nitrogen fertilization and worsen some economically valuable traits, for example, reduce the size of the grain. GRFs (Growth-regulating factors) are a family of genes that encode transcription factors that regulate plant growth and development, organ and tissue formation, and plant response to stress. By inhibiting or overexpressing certain $G R F s$ in rice, it was possible to alter plant height and grain size. Also, GRF4 improves the efficiency of using nitrogen fertilizers in rice. We suggest that by choosing certain allelic variants of GRFs and their combinations with $R h t$ "dwarfing" genes, the use of nitrogen fertilizers can be significantly improved, as well as some of the agronomic characteristics of existing wheat varieties. To identify the $G R F$ alleles that give wheat the desired characteristics, we sequenced several GRFs, developed markers and performed genotyping of a collection of more than 250 Russian common wheat cultivars and found a great allelic diversity of these genes. Then, we performed digital phenotyping of a number of common wheat lines in which GRFs and $R h t$ genes were simultaneously genotyped. Plants were grown indoors under various conditions of nitrogen supply, and their main morphological (height, foliage area, digital biomass, etc.) and spectral (NDVI, etc.) characteristics were non-invasively measured during the first months of development using TraitFinder phenotyping system (Phenospex, Netherlands). The revealed associations between certain combinations of GRFs and $R h t$ genes indicate good prospects for wheat breeding based on specific GRF alleles for the development of new semi-dwarf wheat varieties with improved nitrogen utilization.

Funding: Kurchatov Genomic Center of All-Russia Research Institute of Agricultural Biotechnology, agreement No. 075-15-2019-1667. 